\title{
BMJ Open Impact on cardiovascular risk follow-up from a shift to the CKD-EPI formula for eGFR reporting: a cross-sectional population-based primary care study
}

\author{
Vincent A van Gelder, ${ }^{1}$ Nynke D Scherpbier-de Haan, ${ }^{1}$ Wim J C de Grauw, ${ }^{1}$ \\ Christopher A O'Callaghan, ${ }^{2}$ Jack F M Wetzels, ${ }^{3}$ Daniel S Lasserson ${ }^{4}$
}

To cite: van Gelder VA, Scherpbier-de Haan ND, de Grauw WJC, et al. Impact on cardiovascular risk follow-up from a shift to the CKD-EPI formula for eGFR reporting: a cross-sectional populationbased primary care study. BMJ Open 2013;3:e003631. doi:10.1136/bmjopen-2013003631

- Prepublication history for this paper is available online. To view these files please visit the journal online (http://dx.doi.org/10.1136/ bmjopen-2013-003631).

Received 20 July 2013 Accepted 2 August 2013

\footnotetext{
${ }^{1}$ Department of Primary and Community Care, Radboud University Nijmegen Medical Centre, Nijmegen,

The Netherlands

${ }^{2}$ Nuffield Department of Clinical Medicine, University of Oxford, John Radcliffe Hospital, Oxford, UK ${ }^{3}$ Department of Nephrology, Radboud University Nijmegen Medical Centre, Nijmegen, The Netherlands ${ }^{4}$ Department of Primary Care Health Sciences, University of Oxford, Oxford, UK
}

Correspondence to Vincent A van Gelder; V.vangelder@elg.umcn.nl

\section{ABSTRACT}

Objective: To assess the impact on cardiovascular risk factor management in primary care by the introduction of chronic kidney disease epidemiological collaboration (CKD-EPI) for estimated-glomerular filtration rate (eGFR) reporting.

Design and setting: Cross-sectional study of routine healthcare provision in 47 primary care practices in The Netherlands with Modification of Diet and Renal Disease Study eGFR reporting.

Methods: eGFR values were recalculated using CKD-EPI in patients with available creatine tests. Patients reclassified from CKD stage $3 a$ to CKD stage 2 eGFR range were compared to those who remained in stage $3 a$ for differences in demographic variables, blood pressure, comorbidity, medication usage and laboratory results.

Results: Among the 60673 adult patients (37\% of adult population) with creatine values, applying the CKD-EPI equation resulted in a $16 \%$ net reduction in patients with CKD stage 3 or worse. Patients reclassified from stage $3 a$ to 2 had lower systolic blood pressure (139.7 vs $143.3 \mathrm{~mm} \mathrm{Hg} p<0.0001$ ), higher diastolic blood pressure ( $81.5 \mathrm{vs} 78.4 \mathrm{~mm} \mathrm{Hg} p<0.0001$ ) and higher cholesterol ( $5.4 \mathrm{vs} 5.1 \mathrm{mmol} / \mathrm{L} p<0.0001$ ) compared to those who remained in stage $3 a$. Of those reclassified out of a CKD diagnosis $463(32 \%)$ had no comorbidities that would qualify for annual CVD risk factor assessment and 20 ( $12 \%$ of those with sufficient data) had a EuroSCORE CVD risk $>20 \%$ within 10 years.

Conclusions: Use of the CKD-EPI equation will result in many patients being removed from CKD registers and the associated follow-up. Current risk factor assessment in this group may be lacking from routine data and some patients within this group are at an increased risk for cardiovascular events.

\section{INTRODUCTION}

Chronic kidney disease (CKD) is common and causes substantial morbidity, mortality and healthcare expenditure because it is associated with an increased risk of cardiovascular events as well as progression to end-stage renal

\section{ARTICLE SUMMARY}

Strengths and limitations of this study

- A large population-based study using routinely collected healthcare data with generalisable results.

- Chronic kidney disease (CKD) categories were based on one estimated-glomerular filtration rate measurement.

- Not all patients reclassified out of CKD had data available for cardiovascular risk assessment and the extent of missed high-risk patients with CKD-EPI may be underestimated.

failure. ${ }^{1-3}$ Estimated glomerular filtration rate (eGFRs) are routinely calculated from measured serum creatine values to assess renal function. ${ }^{4}$ The eGFR is central to the classification of CKD into different stages and is used alongside other evidence of kidney disease, such as structural abnormalities on imaging or albuminuria. A key consequence of this staging of CKD is that it is used to guide the management of cardiovascular risk markers, the frequency of follow-up and the need for referral. ${ }^{4}$ In The Netherlands there is guidance for primary care on how to assess and manage cardiovascular risk. ${ }^{5}$

Globally, the standard equation used by healthcare laboratories to calculate eGFR is the four variable Modification of Diet and Renal Disease (MDRD) study equation. ${ }^{6}$ Using pooled data from diagnostic accuracy studies, a newer more accurate equation was developed-the chronic kidney disease epidemiological collaboration (CKD-EPI) equation. ${ }^{7-10}$ Several studies have shown that use of the CKD-EPI equation to calculate eGFR leads to a reduction in CKD diagnoses in younger patients, but an increase in CKD diagnoses in elderly patients. ${ }^{11} 12$ Data from cohort studies and the US health insurance schemes have shown that CKD stage derived from the CKD-EPI formula better 
predicts cardiovascular events and cardiovascular risk than does CKD stage derived from the MDRD equation. ${ }^{13-15}$

The impact on cardiovascular risk follow-up and management in primary care arising from the introduction of the CKD-EPI formula for routine eGFR reporting has not been assessed. We do know that the CKD-EPI formula will change the CKD stage of many patients. ${ }^{11}$ This has important implications as current guideline-driven care pathways emphasise different intensities of monitoring and drug prescribing according to CKD stage. For patients who have their CKD diagnosis removed entirely (by a shift from an MDRD-derived eGFR of $<60$ to a CKD-EPI-derived eGFR of $>60 \mathrm{~mL} / \mathrm{min} / 1.73 \mathrm{~m}^{2}$ in the absence of known albuminuria or other evidence of kidney disease) this will lead to a less intensive treatment of cardiovascular risk factors unless there are comorbid diagnoses such as diabetes mellitus that necessitate enrolment in a cardiovascular risk management programme.

In The Netherlands, all routine chronic disease management is undertaken by primary care physicians in community-based practices, and national guidance on monitoring is available. ${ }^{4}$ Reporting of eGFR with serum creatine in The Netherlands began in 2006 and the EuroSCORE model for cardiovascular risk assessment is currently recommended for use in primary care. ${ }^{5}$ The aim of the study was to determine the cardiovascular risk profile of patients who no longer fulfilled CKD stage 3 criteria when using the CKD-EPI formula for eGFR reporting.

\section{METHOD}

Recruitment of patients

This study used patient data from general practices that participated in a cluster randomised controlled trial on the effect of web-consultation between a general practitioner (GP) and nephrologist on face-to-face referrals-the CONTACT study (Consultation of Nephrology by Telenephrology Allows optimal Chronic kidney disease Treatment in primary care, Netherlands Trial Registration code 2368). The CONTACT study recruited practices during a CKD management course for GPs in the eastern Netherlands. Forty-seven non-academic general practices participated and in the current study the latest data were analysed from their registered populations' electronic medical records between 1 January 2008 and 30 June 2011.

\section{Estimation of renal function}

eGFRs were calculated for patients aged 18 years or older using both the MDRD and CKD-EPI equations. ${ }^{6} 7$ The most recent serum creatine values were selected and were either standardised to isotope dilution mass spectrometry (IDMS) or subject to the appropriate correction factor for laboratories using the Jaffé technique. ${ }^{16}$

\section{Patient characteristics}

Demographic and clinical data including age, sex, albuminuria, comorbidities, medication, blood pressure and lipid levels were extracted from electronic medical records (table 3). Albuminuria was divided into microalbuminuria and macroalbuminuria. Microalbuminuria was defined as a urinary albumin to creatine ratio (ACR) of $2.5-25 \mathrm{mg} / \mathrm{mmol}$ in men and $3.5-35 \mathrm{mg} / \mathrm{mmol}$ in women. Higher ratios were considered to reflect macroalbuminuria. If the ACR was not available we used a urine albumin concentration $>20-200 \mathrm{mg} / \mathrm{L}$ for microalbuminuria and $>200 \mathrm{mg} / \mathrm{L}$ for macroalbuminuria. ${ }^{17}$ Comorbidities were classified using the International Classification of Primary Care (ICPC) codes as a history of the following diseases: cardiovascular disease (CVD) (K74-K77, K89, K90, K92), diabetes mellitus (T90) and hypertension (K86, K87). Anatomical therapeutic chemical (ATC) codes were used to select prescriptions from 2010, and included ACE inhibitors and angiotensin receptor blockers (C09), diuretics (C03) and statins (C10). Blood pressure was reported as the mean of the two most recent measurements. In order to reduce potential under ascertainment bias for chronic disease, if patients were prescribed medications for chronic disease management without an appropriate code, for example, antihypertensive medication without hypertension coded in the medical record, we assumed that the relevant chronic disease was present, even if not coded.

\section{Analysis}

The prevalence of CKD stages derived from the use of the MDRD and CKD-EPI equations was calculated using the size of the registered population aged 18 years and over as the denominator. Demographic and clinical features of patients reclassified from CKD stage 3 a to stage 2 by using the CKD-EPI equation were compared with patients who remained in stage $3 \mathrm{a}$. Next, we specifically described characteristics and cardiovascular risk profile of patients whose CKD stage $3 \mathrm{a}$ was changed to stage 2 who had no diagnosed comorbidity that would otherwise have necessitated their participation in a cardiovascular risk management programme. To assess cardiovascular risk profile we applied the EuroSCORE 10 year risk for CVD. ${ }^{5}$ To ensure conservative estimation we assumed negative smoking status when lacking. Continuous data were compared using $\mathrm{t}$ tests and categorical data were analysed using $\chi^{2}$ tests. Analysis was conducted using SPSS V.20.0 (IBM PASW statistics 20).

\section{RESULTS}

\section{Practice population}

The 47 study practices serve a population of 207469 people of whom 162562 were over 18 years of age. Between 2008 and 2011, 37\% of all adults registered with these practices $(n=60673)$ had their serum creatine measured. In the over 65 age group this figure rose to $71 \%$ (20 959 out of 29591 ).

\section{Study population}

The use of the CKD-EPI equation changed the CKD stage for $20 \%$ of patients $(n=12278)$ with a measured 
Table 1 Reclassification of primary care patients when using CKD-EPI instead of MDRD

\begin{tabular}{|c|c|c|c|c|c|c|c|}
\hline \multirow[b]{2}{*}{ eGFR categories with MDRD (mL/min/1.73 m²) } & \multicolumn{6}{|c|}{ eGFR categories with CKD-EPI (mL/min/1.73m²) } & \multirow[b]{2}{*}{ Total } \\
\hline & $>90$ & $60-89$ & $45-59$ & $30-44$ & $15-29$ & $<15$ & \\
\hline$>90$ & 15195 & 741 & 0 & 0 & 0 & 0 & 15936 \\
\hline 60-89 & 9580 & 27184 & 195 & 0 & 0 & 0 & 36959 \\
\hline $45-59$ & 0 & 1428 & 4338 & 146 & 0 & 0 & 5912 \\
\hline 30-44 & 0 & 0 & 106 & 1345 & 63 & 0 & 1514 \\
\hline $15-29$ & 0 & 0 & 0 & 9 & 290 & 7 & 306 \\
\hline$<15$ & 0 & 0 & 0 & 0 & 3 & 43 & 46 \\
\hline Total & 24775 & 29353 & 4639 & 1500 & 356 & 50 & 60673 \\
\hline
\end{tabular}

CKD-EPI, chronic kidney disease epidemiological collaboration; eGFR, estimated-glomerular filtration rate; MDRD, Modification of Diet and Renal Disease Study.

serum creatine value. This reclassification resulted in a $16 \%$ net reduction in the total number of individuals with CKD stage 3 or worse: 1428 patients were reclassified from stage 3a to stage 2, whereas 195 patients were reclassified from stage 2 to stage 3 a (table 1 ). As a consequence the prevalence of detected CKD stages 3-5 declined from $4.8 \%$ to $4.0 \%$ in the total adult population.

Thirty-two per cent $(n=19235)$ of those who had a serum creatine measurement also had a urine assessment to evaluate albuminuria, and the numbers of patients with different levels of albuminuria are shown in table 2, stratified by eGFR category using both MDRD and CKD-EPI eGFRs. Overall in those tested, the prevalence of microalbuminuria was $12.1 \%(\mathrm{n}=2322)$ and of macroalbuminuria was $1 \% \quad(\mathrm{n}=284)$. Of the patients reclassified from CKD stage 3a to stage 2 eGFR range, albuminuria was tested for in $43 \%(n=617)$ and the prevalence of microalbuminuria was $8.6 \%(\mathrm{n}=53)$ and of macroalbuminuria was $1.5 \%(\mathrm{n}=9)$.

\section{Patients reclassified out of CKD compared to patients remaining in stage $3 a$}

Compared to patients whose CKD stage remained $3 \mathrm{a}$, those who were reclassified to stage 2 range eGFR using the CKD-EPI equation were younger, more were female, with less microalbuminuria, a comparable prevalence of macroalbuminuria, fewer comorbidities and were prescribed fewer antihypertensive and lipid-lowering drugs (table 3). Systolic blood pressure was significantly lower and diastolic blood pressure significantly higher in patients reclassified out of CKD. Total cholesterol and low-density lipoprotein (LDL)-cholesterol were significantly higher in this group.

\section{Patients reclassified out of CKD without other diagnosed comorbidities}

A total of 463 patients (32\% of those reclassified out of CKD) would be removed from a cardiovascular risk management programme as they were not diagnosed with other comorbidities (table 4). Cholesterol measurement was performed in $64 \%$ of patients. The majority of patients had not had an albuminuria assessment. Blood pressure was measured in 218 patients and 111 (51\%) of them had elevated values $\geq 140 / 90 \mathrm{~mm} \mathrm{Hg}$ requiring further monitoring. Similarly, 20 of 172 patients (12\%) with sufficient data to complete cardiovascular risk assessment had a EuroSCORE 10 year CVD risk of $>20 \%$.

\section{Patients reclassified into a CKD diagnosis}

A total of 195 patients were reclassified from stage 2 to stage $3 \mathrm{a}$ CKD, and of these, $166(85 \%)$ had existing comorbidities that would already have identified them for cardiovascular risk assessment. Among the 29 patients who had no identifiable comorbidity, their mean age was 85.4 years (SD 5.1, youngest 76 years) and

Table 2 Numbers of patients with proteinuria according to estimated eGFR category with either MDRD or CKD-EPI in 19235 primary care patients with available results of urine analysis

\begin{tabular}{|c|c|c|c|c|c|c|}
\hline & \multicolumn{6}{|c|}{ eGFR categories $\left(\mathrm{mL} / \mathrm{min} / 1.73 \mathrm{~m}^{2}\right.$} \\
\hline & $>90$ & $60-89$ & $45-59$ & $30-44$ & $15-29$ & $<15$ \\
\hline \multicolumn{7}{|l|}{ MDRD eGFR } \\
\hline Macroalbuminuria & 37 & 97 & 67 & 55 & 21 & 7 \\
\hline Microalbuminuria & 437 & 1140 & 489 & 219 & 35 & 2 \\
\hline No albuminuria & 3016 & 10768 & 2297 & 496 & 51 & 1 \\
\hline \multicolumn{7}{|l|}{ CKD-EPI eGFR } \\
\hline Macroalbuminuria & 43 & 98 & 61 & 53 & 21 & 8 \\
\hline Microalbuminuria & 516 & 1089 & 442 & 226 & 47 & 2 \\
\hline No albuminuria & 4678 & 9617 & 1783 & 490 & 58 & 3 \\
\hline
\end{tabular}


Table 3 Characteristics of patients reclassified out of CKD compared to those who remained in stage $3 a$

\begin{tabular}{|c|c|c|c|c|c|}
\hline Variable & $\begin{array}{l}\text { Remained in } \\
\text { stage } 3 a(N=4338)\end{array}$ & $\begin{array}{l}\text { No of } \\
\text { available data }\end{array}$ & $\begin{array}{l}\text { Reclassified out } \\
\text { of CKD ( }=1428)\end{array}$ & $\begin{array}{l}\text { No of } \\
\text { available data }\end{array}$ & $\begin{array}{l}\mathbf{P} \text { for } \\
\text { comparison }\end{array}$ \\
\hline Age & $74.0(9.5)$ & 4338 & $60.3(9.5)$ & 1428 & $<0.0001$ \\
\hline Female & $61 \% \mathrm{~N}=2654$ & 4338 & $76 \% \mathrm{~N}=1087$ & 1428 & $<0.0001$ \\
\hline Cardiovascular disease & $36 \% \mathrm{~N}=1566$ & 4338 & $18 \% N=250$ & 1428 & $<0.0001$ \\
\hline Hypertension & $57 \% \mathrm{~N}=2463$ & 4338 & $47 \% \mathrm{~N}=666$ & 1428 & $<0.0001$ \\
\hline Diabetes & $26 \% N=1134$ & 4338 & $17 \% \mathrm{~N}=243$ & 1428 & $<0.0001$ \\
\hline None (also excluding albuminuria) & $21 \% \mathrm{~N}=924$ & 4338 & $39 \% \mathrm{~N}=557$ & 1428 & $<0.0001$ \\
\hline Statins & $46 \% N=2000$ & 4338 & $33 \% \mathrm{~N}=475$ & 1428 & $<0.0001$ \\
\hline $\begin{array}{l}\text { ACE inhibitors and/or Angiotensin II } \\
\text { receptor antagonists }\end{array}$ & $54 \% \mathrm{~N}=2349$ & 4338 & $37 \% \mathrm{~N}=533$ & 1428 & $<0.0001$ \\
\hline Diuretics & $43 \% N=1862$ & 4338 & $26 \% N=367$ & 1428 & $<0.0001$ \\
\hline Creatine in $\mu \mathrm{mol} / \mathrm{L}$ & $102(14.3)$ & 4338 & $93(11.4)$ & 1428 & $<0.0001$ \\
\hline Cholesterol/HDL ratio & $4.2(1.4)$ & 3338 & $4.3(1.6)$ & 1118 & 0.020 \\
\hline Total cholesterol in $\mathrm{mmol} / \mathrm{L}$ & $5.1(1.2)$ & 3498 & $5.4(1.1)$ & 1157 & $<0.0001$ \\
\hline $\mathrm{HDL}$ in $\mathrm{mmol} / \mathrm{L}$ & $1.31(0.40)$ & 3454 & $1.36(0.41)$ & 1150 & $<0.0001$ \\
\hline $\mathrm{LDL}$ in $\mathrm{mmol} / \mathrm{L}$ & 3.05 (1.02) & 3451 & $3.31(1.01)$ & 1150 & $<0.0001$ \\
\hline Triglycerides in $\mathrm{mmol} / \mathrm{L}$ & $1.65(0.83)$ & 3458 & $1.64(1.05)$ & 1156 & 0.616 \\
\hline Albumin/creatine ratio* & $1.0(0.9-2.5)$ & 2092 & $0.9(0.7-1.2)$ & 583 & $<0.0001$ \\
\hline Albumin urine in $\mathrm{mg} / \mathrm{L}^{*}$ & $6.0(2.9-17.0)$ & 1174 & $3.0(2.0-8.0)$ & 348 & $<0.0001$ \\
\hline Microalbuminuria & 410 & 2161 & 53 & 617 & $<0.0001$ \\
\hline Macroalbuminuria & 55 & 2161 & 6 & 617 & 0.113 \\
\hline Diastolic blood pressure in $\mathrm{mm} \mathrm{Hg}$ & $78.4(9.4)$ & 3540 & $81.5(9.0)$ & 1043 & $<0.0001$ \\
\hline Systolic blood pressure in $\mathrm{mm} \mathrm{Hg}$ & $143.3(17.5)$ & 3540 & $139.7(15.8)$ & 1042 & $<0.0001$ \\
\hline
\end{tabular}

in the 11 patients with recorded $\mathrm{BP}$, mean values were $137.5 \mathrm{~mm} \mathrm{Hg}$ (SD 13.7)/71.4 $\mathrm{mm} \mathrm{Hg} \mathrm{(5.4).} \mathrm{Of} \mathrm{the} \mathrm{eight}$ patients with sufficient data for CVD risk scoring, six patients were $>20 \%$, although all were outside the age range for accurate scoring and may qualify for high-risk status on age alone.

\section{DISCUSSION}

\section{Principal findings}

Application of the CKD-EPI equation to our study population resulted in a net $16 \%$ decrease (prevalence from
$4.8 \%$ to $4.0 \%$ ) in the total number of individuals with CKD stage 3 or worse based on eGFR criteria. Patients reclassified from stage 3 a to stage 2 were younger, more were female with lower prevalence of comorbidities and a differential effect was seen on blood pressure with lower systolic but higher diastolic mean values. Although $32 \%$ of patients reclassified out of stage 3 a had no documented comorbidity that would entail annual CVD risk factor assessment as part of a chronic disease management programme, an estimated $12 \%$ of these patients did indeed have elevated CVD risk. Not all reclassified patients had an adequate CVD risk factor assessment so

Table 4 Characteristics of patients reclassified out of CKD without diagnosed comorbidity

\begin{tabular}{|c|c|c|}
\hline & Mean (SD) & $\begin{array}{l}\text { No of patients with } \\
\text { available data }\end{array}$ \\
\hline Age & $56.6(10.0)$ & 463 \\
\hline Female & $79 \%$ & 463 \\
\hline Creatine in $\mu \mathrm{mol} / \mathrm{L}$ & 97 (11.9) & 463 \\
\hline Chol/HDL ratio & $4.4(1.6)$ & 285 \\
\hline Total cholesterol in $\mathrm{mmol} / \mathrm{L}$ & $5.8(1.0)$ & 295 \\
\hline $\mathrm{HDL}$ in $\mathrm{mmol} / \mathrm{L}$ & $1.44(0.43)$ & 290 \\
\hline LDL in mmol/L & $3.78(0.89)$ & 289 \\
\hline Triglycerides in $\mathrm{mmol} / \mathrm{L}$ & $1.42(0.76)$ & 289 \\
\hline Albumin/creatine ratio & $0.9(0.5-0.9)$ & 65 \\
\hline Albumin urine in $\mathrm{mg} / \mathrm{L}$ & $2.9(2.0-5.25)$ & 38 \\
\hline Diastolic blood pressure in $\mathrm{mm} \mathrm{Hg}$ & $81.8(9.1)$ & 218 \\
\hline Systolic blood pressure in $\mathrm{mm} \mathrm{Hg}$ & $138.0(16.7)$ & 218 \\
\hline Either systolic or diastolic blood pressure $\geq 140 / 90 \mathrm{~mm} \mathrm{Hg}$ & 111 & 218 \\
\hline EuroSCORE 10 year cardiovascular disease risk $\geq 20 \%$ & 20 & 172 \\
\hline
\end{tabular}


our results may underestimate the scale of this problem, although it is still likely that CKD-EPI reclassification has reduced the number of low-risk patients in stage 3 .

Differences in prevalence of prescribed lipid lowering therapy may explain why higher total cholesterol and LDL levels were seen in patients who were reclassified to a higher eGFR value, above the $60 \mathrm{~mL} / \mathrm{min} / 1.73 \mathrm{~m}^{2}$ cut-off. Higher prevalence of comorbidities, in patients in CKD stage 3 using CKD-EPI estimation, are indications for improved risk factor control for primary and secondary prevention are likely to explain the greater statin prescribing and lower lipid levels in patients retaining a stage 3 CKD diagnosis. However, the differences in blood pressure are not explained by differences in antihypertensive prescribing, due to the differential effect on systolic and diastolic blood pressure.

\section{Strengths and limitations of this study}

One of the strengths of our study is the use of routine general practice data from a large population which enhances the generalisability of our results. In the absence of a screening programme, existing CKD diagnoses are made from clinician-directed testing and so cannot provide true population prevalence. However, we were able to include a large proportion of the (potential) CKD population as creatine results were known in $71 \%$ of patients over 65 years of age. A strength of the analysis of patients reclassified from stage 3 CKD to stage 2 eGFR range is that we made the conservative assumption that patients prescribed medication used to treat comorbidities did indeed have that comorbidity, even if it was not coded in the medical record. This will have the effect of reducing the number of patients in the subgroup without comorbidity reclassified out of a CKD diagnosis, and the impact that we did find is likely to underestimate the effect of reclassification. Furthermore, our study accurately reflects the changes in CKD stage and status that will arise from use of the CKP-EPI formula in current clinical practice with clinician-directed testing.

A limitation of this study is that our classification of patients was based on a single creatine result, whereas guidelines recommend two measurements $\geq 3$ months apart before making a diagnosis. However, this is unlikely to affect the proportional changes seen to a great extent given the size of our dataset and is in line with the approach used in most CKD studies. ${ }^{11} 12$ 18-20 Albuminuria and cardiovascular risk factor assessment were incomplete and this highlight an area for quality improvement in general practice. However, despite the low usage of albuminuria assessment, our estimates of the gains and losses in CKD diagnoses are likely to be reliable. We did not have ethnicity data and assumed our population to be Caucasian. This assumption was based on ethnicity data from the Statistics Netherlands that shows $92 \%$ of the population is Caucasian in the eastern Netherlands. ${ }^{21}$ Both MDRD and CKD-EPI equations estimate true GFR and are therefore prone to some degree of error.

\section{Comparison with existing literature}

In other studies, a similar decrease in CKD prevalence was found: from $4.2 \%$ to $3.9 \%$ and $4.9 \%$ to $4.4 \%$ in two large adult UK population-based studies, ${ }^{18}$ and from $5.41 \%$ to $4.80 \%$ in the Quality Improvement in CKD trial. ${ }^{22}$ Also, CKD stage alteration in one-fifth of all patients with a creatine measurement by use of the CKD-EPI equation is a figure consistent with previous estimates. ${ }^{11}$

Cohort studies stratifying patients at baseline with reclassified CKD stages using CKD-EPI eGFRs report lower cardiovascular events in these patient groups during follow-up, ${ }^{13-15}$ suggesting that overall, risk prediction is improved. Two studies that analysed the characteristics of patients reclassified out of a CKD diagnosis using CKD EPI are in agreement with our results, finding that these patients were predominantly women, of younger age and with less diabetes compared to those whose CKD stage remained unchanged. ${ }^{19} 23$ White $e t a l^{19}$ did not report diastolic blood pressure or LDL-cholesterol levels, but found that those reclassified out of CKD had lower systolic blood pressure, similar total cholesterol levels and lower triglycerides levels and a lower 10-year CVD risk. Although we also found lower systolic blood pressure levels, we found higher total (and LDL) cholesterol and no reduction in triglyceride levels. The differential effect on systolic and diastolic blood pressure may be explained by the fact that the reclassified patients in our study had a younger mean age. Diastolic blood pressure has been shown to be high in early middle age and then fall in older age as systolic blood pressure increases. ${ }^{24}$ Patients with a CKD-EPI eGFR sufficiently higher than their MDRD eGFR to lose their CKD diagnosis are young enough to exhibit this effect.

Korhonen et $a l^{20}$ found no significant differences in cardiovascular risk factors between those reclassified and those remaining in the same CKD stage when the CKD-EPI formula was used, but the study size $(n=1747)$ was probably too small to detect the differences we identified. No previous study has examined medication use.

\section{Meaning of the study}

Introduction of CKD-EPI to calculate eGFR can be expected to lower primary care workload and reduce treatment and costs in patients who overall have low cardiovascular risk. While the CKD-EPI equation shows greater calibration than the MDRD equation in that it more accurately stratifies patients in terms of their cardiovascular risk, we have found that by using routinely collected data in primary care, patients reclassified out of CKD had a more favourable cardiovascular profile in terms of age and comorbidity compared to patients who remained in stage $3 \mathrm{a}$. However, there is a small group of patients with elevated cardiovascular risk who will no longer be detected and managed.

\section{Unanswered questions and future research}

Uncertainty remains about the impact on patient cardiovascular follow-up in those who lose their CKD diagnosis 
with use of the CKD-EPI formula. Although fewer cardiovascular events are seen in patients reclassified from retrospective observational data, ${ }^{13-15}$ their care would have been guided by MDRD staging rather than the less severe CKD stage seen with CKD-EPI. Prospective follow-up studies are therefore required to appropriately determine the impact of CKD-EPI GFR estimation on cardiovascular events.

Current guidelines apply a fixed eGFR threshold to define CKD with the result that some patients will not be diagnosed with CKD in the presence of reduced renal function for their age group. This effect may be particularly relevant in younger patients, who may for example, have a 'normal' eGFR of $80 \mathrm{~mL} / \mathrm{min} / 1.73 \mathrm{~m} 2$ which is 'normal' according to Dutch guidelines ${ }^{4}$ yet it is significantly lower than the average GFR in people of a similar age. ${ }^{25}$ It may be more appropriate to report the statistical deviation of eGFR values from age-matched population mean and to highlight those that lie below a given percentile for further assessment and cardiovascular risk assessment. The CKD-EPI equation would make such an approach more feasible since it can be used to accurately report on eGFR $>60 \mathrm{~mL} / \mathrm{min} / 1.73 \mathrm{~m}^{2 .}{ }^{8}$ Further research is therefore needed to determine the feasibility of agematched eGFR reporting to guide patient management.

Contributors DSL, CAO'C, NSdH conceived the study. VvG and DSL undertook the analyses. VvG and DSL drafted the manuscript and all authors contributed to study design, data interpretation, revisions and approved the final version.

Funding The Dutch Kidney Foundation funded the study (Nierstichting Nederland). The Department of Primary and Community Care received a non-conditional grant from Amgen. DSL and CAO'C are supported by the National Institute for Health Research (NIHR) Oxford Biomedical Research Centre Programme. The views expressed are those of the authors and not necessarily those of the NHS, the NIHR or the Department of Health.

Competing interests None.

Ethics approval Medical Research Ethics Committee Arnhem/Nijmegen registration number 2010/187. This study was performed according to the Code of Conduct for Health Research which has been approved by the Data Protection Authorities for conformity with the applicable Dutch privacy legislation.

Provenance and peer review Not commissioned; externally peer reviewed.

Data sharing statement No additional data are available.

Open Access This is an Open Access article distributed in accordance with the Creative Commons Attribution Non Commercial (CC BY-NC 3.0) license, which permits others to distribute, remix, adapt, build upon this work noncommercially, and license their derivative works on different terms, provided the original work is properly cited and the use is non-commercial. See: http:// creativecommons.org/licenses/by-nc/3.0/

\section{REFERENCES}

1. Coresh J, Selvin E, Stevens LA, et al. Prevalence of chronic kidney disease in the United States. JAMA 2007;298:2038-47.

2. Go AS, Chertow GM, Fan D, et al. Chronic kidney disease and the risks of death, cardiovascular events, and hospitalization. $N$ Engl J Med 2004;351:1296-305.

3. Weiner DE, Tighiouart H, Amin MG, et al. Chronic kidney disease as a risk factor for cardiovascular disease and all-cause mortality: a pooled analysis of community-based studies. J Am Soc Nephrol 2004;15:1307-15.
4. De Grauw WJC, Bilo HJG, Faber EF, et al. Landelijke transmurale afspraak chronische nierschade. Huisarts Wet 2009;52:586-97.

5. NHG. Standaard Cardiovasculair risicomanagement (eerste herziening). Huisarts Wet 2012;55:14-28.

6. Levey AS, Bosch JP, Lewis JB, et al. A more accurate method to estimate glomerular filtration rate from serum creatinine: a new prediction equation. Modification of Diet in Renal Disease Study Group. Ann Intern Med 1999;130:461-70.

7. Levey AS, Stevens LA, Schmid $\mathrm{CH}$, et al. A new equation to estimate glomerular filtration rate. Ann Intern Med 2009;150:604-12.

8. Stevens LA, Schmid CH, Greene T, et al. Comparative performance of the CKD Epidemiology Collaboration (CKD-EPI) and the Modification of Diet in Renal Disease (MDRD) Study equations for estimating GFR levels above $60 \mathrm{~mL} / \mathrm{min} / 1.73 \mathrm{~m} 2$. Am J Kidney Dis 2010;56:486-95.

9. Becker BN, Vassalotti JA. A software upgrade: CKD testing in 2010. Am J Kidney Dis 2010;55:8-10.

10. Madero M, Sarnak MJ. Creatinine-based formulae for estimating glomerular filtration rate: is it time to change to chronic kidney disease epidemiology collaboration equation? Curr Opin Nephrol Hypertens 2011;20:622-30.

11. O'Callaghan CA, Shine B, Lasserson DS. Chronic kidney disease: a large-scale population-based study of the effects of introducing the CKD-EPI formula for eGFR reporting. BMJ Open 2011;1: e000308.

12. Van den Brand JA, Van Boekel GA, Willems HL, et al. Introduction of the CKD-EPI equation to estimate glomerular filtration rate in a Caucasian population. Nephrol Dial Transplant 2011;26:3176-81

13. Matsushita K, Selvin E, Bash LD, et al. Risk implications of the new CKD Epidemiology Collaboration (CKD-EPI) equation compared with the MDRD Study equation for estimated GFR: the Atherosclerosis Risk in Communities (ARIC) Study. Am J Kidney Dis 2010;55:648-59.

14. Shafi T, Matsushita K, Selvin E, et al. Comparing the association of GFR estimated by the CKD-EPI and MDRD study equations and mortality: the third national health and nutrition examination survey (NHANES III) examination survey (NHANES III). BMC Nephrol 2012;13:42.

15. Matsushita K, Mahmoodi BK, Woodward M, et al. Comparison of risk prediction using the CKD-EPI equation and the MDRD study equation for estimated glomerular filtration rate. JAMA 2012;307:1941-51.

16. Levey AS, Coresh J, Greene T, et al. Expressing the Modification of Diet in Renal Disease Study equation for estimating glomerular filtration rate with standardized serum creatinine values. Clin Chem 2007:53:766-72.

17. Ozyilmaz A, Bakker SJ, De Zeeuw D, et al. Selection on albuminuria enhances the efficacy of screening for cardiovascular risk factors. Nephrol Dial Transplant 2010;25:3560-8.

18. Carter JL, Stevens PE, Irving JE, et al. Estimating glomerular filtration rate: comparison of the CKD-EPI and MDRD equations in a large UK cohort with particular emphasis on the effect of age. QJM 2011;104:839-47.

19. White SL, Polkinghorne KR, Atkins RC, et al. Comparison of the prevalence and mortality risk of CKD in Australia using the CKD Epidemiology Collaboration (CKD-EPI) and Modification of Diet in Renal Disease (MDRD) Study GFR estimating equations: the AusDiab (Australian Diabetes, Obesity and Lifestyle) Study. Am J Kidney Dis 2010;55:660-70.

20. Korhonen PE, Kautiainen $\mathrm{H}$, Jarvenpaa $\mathrm{S}$, et al. Time to change the glomerular filtration rate estimating formula in primary care? Eur $\mathrm{J}$ Intern Med 2012:23:355-7.

21. Netherlands S. [10/05/2013]. http://statline.cbs.nl/StatWeb/ publication $/$ ?VW $=$ T\&DM $=S L N L \& P A=37713 \& D 1=0$ $8 \& D 2=0 \& D 3=0,3 \& D 4=2 \& D 5=12-15 \& H D=130213-$ $1715 \& \mathrm{HDR}=\mathrm{T} \& \mathrm{STB}=\mathrm{G} 1, \mathrm{G} 2, \mathrm{G} 3, \mathrm{G} 4$

22. De Lusignan $\mathrm{S}$, Tomson $\mathrm{C}$, Harris $\mathrm{K}$, et al. Creatinine fluctuation has a greater effect than the formula to estimate glomerular filtration rate on the prevalence of chronic kidney disease. Nephron Clin Pract 2011:117:c213-24.

23. Schold JD, Navaneethan SD, Jolly SE, et al. Implications of the CKD-EPI GFR estimation equation in clinical practice. Clin J Am Soc Nephrol 2011:6:497-504.

24. Wright JD, Hughes JP, Ostchega $Y$, et al. Mean systolic and diastolic blood pressure in adults aged 18 and over in the United States, 2001-2008. Natl Health Stat Rep 2011;4:1-22.

25. Wetzels JF KL, Swinkels DW, Willems HL, et al. Age- and gender-specific reference values of estimated GFR in Caucasians: the Nijmegen Biomedical Study. Kidney Int 2007;72:632-7. 